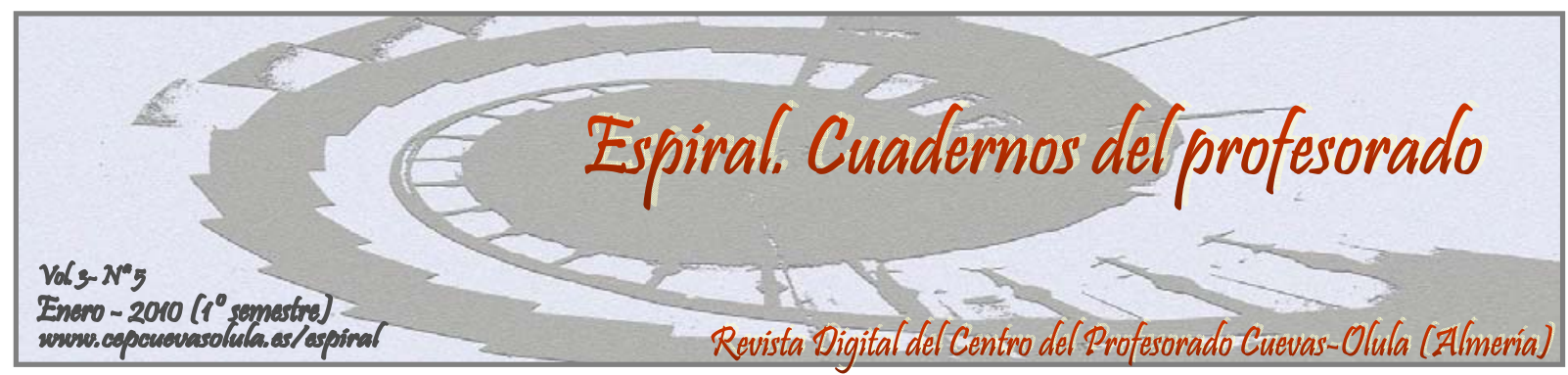

\title{
LOS MUSEOS DIDÁCTICOS Y EL MUSEO MUSICAL: UN ESTUDIO CONCEPTUAL
}

\section{MUSEUMS AND MUSEUM MUSICAL LEARNING: A CONCEPTUAL STUDYTHE}

\author{
Alejandro Vargas Serrano \\ Conservatorio Elemental de Música de Olula del Río, Almería (España)
}

RESUMEN: Es frecuente que dentro de la actividad docente realicemos visitas a Museos o Bienes Patrimoniales, ya sea con nuestros alumnos o de manera privada. Cuando debemos ir con los alumnos, a menudo nuestra máxima prioridad y atención va destinada a supervisar la actividad más que a propiciar aprendizajes en nuestros alumnos. Recientemente, hemos vivido esta experiencia. En una visita concreta a uno de los museos de Andalucía lo experimentamos. Es por ello que en este artículo pretendemos realizar un pequeño estudio conceptual de la terminología que va asociada a los museos didácticos, partiendo desde el análisis del concepto Museo, pasando por el museo didáctico - museo musical y sus características, para concluir en el planteamiento necesario de realizar estudios de público. La distinción entre las diferentes tipologías de museos, así como específicamente entre los diferentes museos musicales según su finalidad, nos puede ayudar no solo en nuestra labor docente para con nuestro alumnado, sino además en nuestra propia formación intelectual.

Palabras clave: museo didáctico, TIC, museo musical.

ABSTRACT: It is common within the teaching activity to museums or heritage asset, either with our students or privately. When we go with students, this is the reason our highest priority and attention is directed to monitor the activity rather than to promote learning in our students. We love recently had this experience. We experience it in a specific visit to one of the museums in Andalusia. In this article we intend to conduct a small study of the conceptual terminology that is associated with educational museums, starting from the museum concept analysis, through the educational museum - museum and features music, concluding with the approach required to conduct audience surveys. The distinction between different types of museums, and specifically between the various museums music for their purpose, can help us not only in our work with students, but also in our own intellectual formation.

Key words: educational museum, ICT, music museum.

Vargas, A. (2010). Los museos didácticos y el museo musical: un estudio conceptual. Espiral. Cuadernos del Profesorado [en línea], 3(5), 44-50. Disponible en: http://www.cepcuevasolula.es/espiral. 


\section{1.- INTRODUCCIÓN. IDEALISMO.}

Ya que un museo es el lugar donde se guardan los objetos que pueden llegar a definir una cultura, a continuación brevemente vamos a explicar el concepto de cultura y como interpretar una nueva idea (en justificación a la idea de museo didáctico).

Cuando una idea surge en el plano intelectual, se comienza un proceso en el que la idea se proyecta a diferentes ámbitos. Se comienza por su exploración, en la que se llegan a resolver problemas fundamentales. En ocasiones son tantos los problemas que resuelve que se cree que pueda ser la provocadora de un nuevo sistema de análisis. Pero cuando esta idea pasar de ser nueva y ya se ha debatido lo suficiente sobre ella, se hacen razonamientos más conscientes y equilibrados que permiten, a la hora de su aplicación sea de tal forma que por un lado, se razonen los problemas que la nueva idea es capaz de generar, y por otro, que su aplicación sea consciente $y$ se utilice para resolver problemas que realmente esa idea sea capaz de resolver. Se trata de limitar las aplicaciones de las grandes ideas. Es este quizás el método que la antropología utiliza.

El concepto de cultura se ve en mayor o menor medida especializado según las ideas que se utilicen para su definición.

\section{2.- CONCEPTO DE MUSEO.}

El término museo proviene del latín "museum" y este a su vez del griego "mouseion" que significa casa de las musas. Aunque nos puede dar una idea aproximada, no se proyecta lo que actualmente se entiende como tal. Una definición actual y más acorde con las características de los museos, después de diferentes modificaciones, es la elaborada por el ICOM (International Council of Museums). "El museo es una institución permanente, sin finalidad lucrativa, al servicio de la sociedad y de su desarrollo, abierto al público, que adquiere, conserva, investiga, comunica y exhibe para fines de estudio, de educación y deleite, testimonios materiales del hombre y su entorno". Según el ICOM las tipologías museísticas son las siguientes:

- Museos de arte: arqueológicos, de Bellas artes, de Arte contemporáneo y Artes decorativas.

- Museos Generales especializados monográficos y mixtos: ciudades-museo, mu- seos al aire libre, jardines, reservas, y parques naturales.

- Museos de historia: variedad de los museos históricos, Museos militares y navales.

- Museos de etnología, Antropología y Artes populares.

- Museos de Ciencias naturales.

- Museos científicos y de técnica industrial.

Pero dentro de esta concepción y estas tipologías hay algo más. El propio ICOM ha desarrollado nuevas propuestas de museo en las que este pasa de ser un lugar de actividad totalmente pasiva por parte del visitante. Las nuevas propuestas giran en torno a la introducción de nuevos valores en el público. Lo que se trata es de dotar al visitante de protagonismo, de relacionar el museo pedagógicamente con el visitante. Son varios los elementos que se pueden utilizar, y de hecho algunos museos están dotados ya de fichas, talleres y realización de diferentes actividades, que permitan una relación más directa del público con el museo. Existen algunos museos que llegan a tener incluso un departamento pedagógico encargado de estas cuestiones.

\section{3.- OBJETO DE LOS MUSEOS.}

Podríamos decir que las actuales teorías consideran musealizable todo objeto que pertenece a la llamada cultura material, la cual está constituida por objetos a los cuales les suponemos un valor de documento, los suponemos portadores de información. Ahora bien, todos los objetos en mayor o menor medida son portadores de información, sobre todo con el paso del tiempo por ellos. Todo objeto que pertenezca a una cultura material a partir de él se puede conocer las razones que motivaron su aparición. Los objetos por tanto, pueden, contarnos la historia de una determinada cultura. Además si la cultura no tiene una tradición representada a través de la escritura, el objeto material adquiere mayor importancia. El objeto es un testigo directo y "objetivo" de la historia de la cultura.

Por lo general se considera como objeto musealizable aquel que destaca por su valor estético y rareza. Esto es, cuando un objeto deja de entenderse como tal para pasar a ser una obra de arte. Es entonces cuando a la obra de arte no solo se la dota de un valor estético sino que también de un valor social. Además estos valores generalmente suponen una exaltación del objeto. 
En este sentido son varias las corrientes de pensamiento que han reaccionado (por ejemplo los -ismos de principios del siglo XIX).

Una nueva concepción del museo ha de representar un papel más cercano al visitante. Este es el caso de los eco-museos, cuyos objetos de estudio suelen ser ropas, instrumentos y lo que hoy llamaríamos enseres que identifican una época determinada en un determinado territorio. El museo sirve en este caso como intercesor para que el visitante a través de él conozca esa época en un determinado territorio. Esta comunicación el museo la puede establecer directamente exponiendo los objetos, o con ayuda a su identificación con paneles que expliquen por qué ese objeto se encuentra allí.

\section{4.- MUSEO DIDÁCTICO.}

El museo didáctico surge dentro la evolución del concepto tradicional de museo. Es el momento en el que se tiende hacia una mayor intencionalidad comunicativa. El museo tradicional expone los objetos para ser contemplados, de esta forma el visitante sólo tiene en cuenta el valor estético del objeto. En la mayoría de los casos, para ir más allá el visitante debía de tener una serie de conocimientos que le permitiesen valorar de acuerdo a una cultura determinada el objeto expuesto, o en su caso, la obra de arte. Es por ello que hacia mediados del siglo $\mathrm{XX}$ se intenta una socialización del museo.

Esto no surgirá espontáneamente sino que gracias a los acontecimientos sociales y a la implantación de la nueva democracia el museo poco a poco se fue socializando. Evidentemente la historia nos enseña que en no todos los países se lleva a cabo. Mientras algunos muestran su preferencia por una educación elitista diseñada y establecida para unos pocos, otros intentaron, debido generalmente a los avances técnicos y a la necesidad de determinados conocimientos para determinados trabajos, implantar un sistema de educación más accesible y cercana a la mayoría.

El conflicto fue denominado como "crisis mundial educativa" por Philip H. Coombs. En su estudio refleja la necesidad de ampliar los medios educativos fuera de las escuelas. Su aportación al hecho educativo fue de vital importancia, términos como educación informal (adquisición de conocimientos durante toda nuestra vida) y educación no formal (organización sistemática de una actividad para facilitar el aprendizaje a grupos determinados de la sociedad), supusieron un avance dentro de la evolución del proceso educativo. El concepto de educación fue ampliado de tal forma que se formularon nuevas formas de aprendizaje, más complejas y efectivas.

En el museo didáctico, a diferencia del museo contemplativo (solo muestra los objetos) o del museo informativo (muestra los resultados de un proceso de investigación), el visitante deja de tener una actitud pasiva. El museo didáctico es el que enseña a aprender a través del análisis de la cultura ofreciendo al visitante los objetos, la información y los medios necesarios para que el visitante elabore sus propias interpretaciones. De esta forma cada visitante adquiere su propia interpretación.

A pesar de que el museo didáctico es de gran interés sociocultural, su propio carácter hace que se pueda llegar a dudar que este tipo de museos entren dentro de la definición que antes mencionamos. Los objetos en un museo didáctico se deben tocar sentir, algo que es impensable dentro de un museo convencional.

Los museos tradicionales han ido incorporando poco a poco nuevos materiales para que el visitante comprenda la obra, aparte de por su valor estético. Estos materiales suelen ser fichas didácticas, trípticos o cuadernos monográficos dedicados a diferentes tipos de visitantes, generalmente destinado a estudiantes de diferentes tipos de nivel educativo.

El museo didáctico se dirige a toda la sociedad, aunque los existentes dirigen su atención hacia el público joven, generalmente niños y adolescentes. La organización es distinta a la de un museo convencional, ya que este tipo de museos ha de contar con una programación determinada junta con una organización de las presentaciones.

Las razones principales por las que el museo didáctico se proyecta para este tipo de visitantes (niños y adolescentes), pueden sen las siguientes.

- El museo cuenta con unos recursos limitados, con lo que una representación para cada tipo social de individuos supondría un coste en material y personal excesivo.

- Los niños son los futuros adultos por lo que han de ser conscientes de los nuevos avances e ideologías.

- Es más fácil formar ideas que cambiar unas ya establecidas, es decir el niño se encuentra en disposición de aprender mientras que el adulto no siempre lo está. 
Este tipo de museos aunque pensados para este tipo de visitantes, no están dedicados exclusivamente a ellos. Es normal la visita de público más maduro que disfruta también de este tipo de museos. Se puede llegar a decir que los adolescentes serían el público real, mientras que los adultos serían el público potencial.

En el museo didáctico los objetos se exponen siguiendo unos determinados criterios expositivos. Además de una disposición lógica y ordenada. De esta forma los conocimientos que el museo tiene la intención de transmitir se ven condicionados por diferentes factores, entre los que destacan:

- Los derivados de las teorías pedagógicas vigentes en el momento de la exposición.

- Los derivados del entorno, edad, condicionantes varios del público al que se dirige.

- Los derivados de su intencionalidad comunicativa, ya que son el medio del museo para expresar sus objetos.

\section{5.- MUSEOS Y EDUCACIÓN.}

Las teorías pedagógicas que en España desembocaron en la implantación de la LOGSE, y las sucesivas leyes actuales, son las mismas que intervienen en los criterios expositivos del museo. Este soporte teórico se basa en la teoría cognitivista de Bruner, la psicología educativa de Vigotsky y en la psicología genética de Piaget. Será él quien establezca las etapas de formación de la mente en la evolución de los niños.

La primera de estas etapas la sitúa entre las edades de 7 a 11 años. Las define como el periodo de las operaciones concretas. En esta etapa los niños centran su análisis en la asimilación de las distintas cualidades de los objetos mediante un proceso lógico de relaciones.

La segunda de estas etapas está comprendida entre los 11 a 15 años. Es, para Piaget, la etapa de las operaciones formales. En esta etapa se desarrolla la capacidad en el niño para realizar procesos de investigación completos.

Estas dos franjas de edad son a las que se destinan principalmente los museos didácticos. Pero en ellos no se encuentra una separación para que cada edad tenga un tipo de exposición. Para un mejor aprendizaje la especialización de los contenidos dentro del museo debería ser la constante en la organización de las exposiciones.

$\mathrm{Al}$ menos en un museo didáctico la finalidad comunicativa es la detonante en los factores que condicionan la exposición en el museo didáctico.
Su función principal es la de enseñar a aprender, esto es, que el visitante alcance gracias a procesos constructivistas basándose en hipótesis, determinados conocimientos según los datos que se ofertan. El criterio expositivo en este tipo de museos, ha de ser de acuerdo a un estudio científico previo a la exposición que sugiera el mismo estudio que se llevó a cabo para determinar el descubrimiento. Esto será posible gracias a la disposición lógica de todos los datos.

Ya que las exposiciones didácticas se basan en el conocimiento, y por lo tanto, en gran medida en la ciencia, su carácter ha de ser necesariamente renovable, es decir temporal. Este aporte dinámico al museo hace que se lleve a cabo un continuo proceso de reciclaje y estudio.

Junto al carácter comunicativo y de enseñanza del museo didáctico, se inscribe también el carácter lúdico. El juego ha de estar presente igualmente. Siempre desde luego bajo un control razonado. El juego es el medio por el que los niños entran en contacto con los objetos. Constituye un elemento clave dentro del proceso enseñanza-aprendizaje. Pero no conviene abusar dando lugar a que solamente se trate de exposiciones con carácter lúdico.

Los criterios que rigen el montaje de una exposición deberán depender siempre del carácter de los objetos que se exponen. Estos no serán seleccionados según criterios estéticos, sino más bien según a criterios didácticos. Es decir, los objetos que se deben elegir para una exposición de un museo didáctico han de ser documentos que transmitan un significado concreto. No son pues, obras de arte lo que un museo didáctico muestra, sino que, los objetos que allí se exponen transmiten información fundamentalmente.

Debido a los problemas de relación con el público y de los extensos campos y funciones que se desarrollan en un museo didáctico, existe toda una política de comunicación que se encarga de definir el posicionamiento del museo. Los principales puntos de los que tratan este tipo de política son las siguientes:

- Relación entre el museo y los medios de comunicación.

- Clases y modelos de materiales a difundir.

- Identificación del público con el que se quiere comunicar.

- Niveles a los que se quiere llevar esa comunicación.

- Formas de comunicación y lenguaje. 
- La imagen que se quiere transmitir y el diseño.

- Los recursos disponibles y las prioridades.

Dentro de la organización comunicativa del museo uno de los puntos importantes es el de programación educativa. Se trata de una comunicación directa y personal que ofrece una visión dinámica de la exposición. En esta programación los elementos que permiten esa comunicación, no pueden fallar dentro del museo didáctico. Si los elementos comunicativos fallan dentro de un museo clásico, no importa demasiado ya que, estos no condicionan directamente la exposición. En cambio si en un museo pensado con fines didácticos, los elementos comunicadores fallan, el museo no logra esa función de aprendizaje que antes señalábamos.

\section{6.- APLICACIONES DE LAS NUEVAS TECNOLOGÍAS.}

La incorporación de las nuevas tecnologías a la vida de los museos ha revolucionado muchos de los aspectos de sus formas de comunicación, este hecho es especialmente destacable para el museo didáctico que acostumbra a aprovechar al máximo estas innovaciones.

Generalmente uno de los usos para los que se utilizan los medios audiovisuales dentro de un museo es destinado, para que el visitante conozca lo que se va encontrar en la exposición previamente. Lo cual hace que el visitante ya obtenga una predisposición de lo que va a ver, oír y sentir. El elemento de sorpresa no tiene porqué perderse.

En muchos casos el museo no expone todo lo que tiene debido a la falta de espacio o de climatización. Por ello los medios audiovisuales permiten ver las colecciones enteras y todo el muestrario que el museo posee.

Generalmente los medios que han utilizado para difundir este tipo de información, por parte del museo han sido dossieres, panfletos explicativos..., u otras fórmulas parecidas. Con las nuevas tecnologías se puede optar por diferentes perspectivas más allá de las que un folleto hace ver. En el caso de una exposición virtual, el visitante puede seleccionar aquello que más le interesa, puede optar por una visualización determinada de la información que el museo ofrece y no tiene que esperar o cumplir horarios. En muchos casos las nuevas tecnologías ofrecen

Espiral. Cuadernos del Profesorado. ISSN 1988-7701

2010, vol. 3, no 5, pp. 44-50 todo un nuevo campo que permite un mayor conocimiento del objeto.

La informática y su aplicación al museo ofrecen todo un sin fin de posibilidades para contemplar y exhibir las exposiciones. Es frecuente encontrar CD-roms en los que se incluyan las obras de las que el museo está dotado.

Internet ofrece aún más, ya que poder visitar los museos desde cualquier lugar y sin necesidad de desplazarse hasta su emplazamiento, es algo que enriquece y abre más las puertas para que el público en general acceda.

\section{7.- NECESIDADES ARQUITECTÓNICAS.}

Ante la poca difusión de los museos didácticos, los criterios arquitectónicos han de estar condicionados por los elementos que los constituye, como son, el tipo de público, la colección del museo, los criterios expositivos...

Este tipo de museos, como antes señalábamos es un tipo de museos relativamente jóvenes. La nueva concepción de museo surge aproximadamente a mediados del siglo XX. Además el empleo de las nuevas tecnologías, así como la importancia de la comunicación, hacen necesaria un tipo de construcción moderna y acorde a la época en la que estos museos surgen.

Los museos didácticos necesitan un espacio interior adaptado a las necesidades que presentan. Normalmente hay que tener en cuenta que muchas de las exposiciones que albergan este tipo de museos necesitan un determinado espacio, donde se puedan instalar por ejemplo grandes pantallas de televisión.

Las barreras arquitectónicas tienden a desaparecer en este tipo de museos, ya que la versatilidad necesaria para albergar diferentes tipos de estructuras expositivas y su duración, muchas veces corta en el tiempo, hacen que el espacio necesario sea lo menos condicionado por la arquitectura.

No solamente se debe de tener en cuenta las necesidades de la propia exposición. Además se debe de tener en cuenta las necesidades del visitante. Lo aconsejable es un espacio acorde con los usuarios que en estos museos suele ser niños (de estatura menor a la de los adultos que programan la exposición).

\section{8.- MUSEOS MUSICALES.}

La función que en la actualidad desempeñan la mayoría de museos musicales, es dispar. La 
mayoría de ellos cuenta con cantidades enormes de instrumentos musicales. La selección de estos instrumentos, como casi siempre, es llevada a cabo por los comisarios de las exposiciones. El enfoque general suele ser el expositivo, aunque cada son más frecuentes los museos que cuentan con enfoques didácticos.

Los instrumentos expuestos no solamente poseen un valor patrimonial sino que han de ser analizados desde un punto de vista educativo. No solamente enfocado a los escolares sino al público en general. El escolar en cierto sentido, dependiendo del enfoque dado a la visita aprende un número determinado de contenidos. La aplicación de las plantillas antes comentadas, en muchos casos suele ser casi anecdótica y la implicación por parte del profesorado antes, durante y después de la visita, no siempre es la acertada para construir aprendizajes en sus alumnos. El museo musical tiene un enorme potencial didáctico. En el caso de niños, el juego, las nuevas tecnologías el poder tocar y experimentar va a suponer que las visitas sean dinámicas, divertidas, enriquecedoras y sobre todo van a constituir aprendizajes significativos en los alumnos.

Un ejemplo es el museo interactivo de Música de Málaga. Inaugurado en el año 2002, cuenta con una gran cantidad de instrumentos de diferentes países y épocas. En este museo prima el uso del ordenador y de las denominadas nuevas tecnologías, permitiendo la interactividad. El propio museo cuenta con una serie de talleres y actividades para escolares, grupos, familias o adultos. Otro museo que sigue esta línea aunque de forma diferente es el museo de Ureña que cuenta con un departamento pedagógico encargado de organizar módulos didácticos para realizar con anterioridad a la visita, durante la visita y a posteriori.

En cambio y pese a que cada vez más los museos van optado por la organización didáctica la mayoría de museos de música, optan por el valor expositivo. En España es el caso de museos como el Museo Nacional de Etnología de Madrid, el Museo Municipal de Música de Barcelona, el Museo del Palacio de la Música, y tantos otros. En Europa, entre otros destacan; en Bélgica, el Museo del Conservatorio de Música de Bruselas (cuenta con más de 4000 instrumentos); en Alemania (con gran calidad de sus instrumentos y bienes patrimoniales musicales), el Museum für Volkerkunde
Staaliche (Berlín); en Viena el Museum für Volkerkunde...

\section{9.- CONCLUSIONES: LOS ESTUDIOS DE PÚBLICO.}

Los estudios sobre visitantes a museos y la evaluación de la recepción de las exposiciones tiene cada día una mayor importancia para la gestión del patrimonio cultural y la formulación de las políticas que la orientan. Consideramos que la contribución disciplinar de la antropología resultaría valiosa a la hora de conocer las representaciones que los distintos sectores sociales tienen de la institución 'museo' y de sus prácticas de interpretación del patrimonio cultural.

Una perspectiva desde la antropología del estudio de visitantes podría proporcionar elementos para poner en evidencia que no sólo el público sino también los políticos, funcionarios técnicos, funcionarios burocráticos, sustentan sus prácticas en modelos de representación estereotipados, cuyo conocimiento es necesario para formular una política de gestión cultural y planificar su implementación y evaluación. Los museos, pero fundamentalmente las áreas políticas de las cuales dependen, podrían hacer una utilización de este aporte para registrar y comprender las discrepancias entre la imagen que los museos tienen de sí mismos, la que sostienen quienes diseñan las políticas culturales y la construida por los diversos públicos que se supone son sus destinatarios.

Los estudios del publico tienden a poner en evidencia que una exposición es un medio de comunicación, es una propuesta multimedia conformado no solo por el patrimonio cultural sino por la interacción de la experiencia del visitante junto con la concurrencia del espacio, los soportes, la iluminación y por la cual se puede "navegar" diseñando diferentes rutas en diferentes momentos y circunstancias. Los museos son espacios culturales donde el publico utiliza su tiempo libre, a los cuales acude a aprender, a divertirse, a cumplir un acto de iniciación ritual y en donde se da una actividad socializante, se le presentan nuevas formas de percibir el mundo y de repensar los lazos de continuidad con el pasado histórico de su comunidad de pertenencia.

En general se observan dos principales tendencias en este campo. Los estudios del publico a museos como estudios críticos de las praxis de las institucionales y la que considera el 
estudio de los visitantes como un servicio permanente que el museo debe brindar al publico. En ambos casos sería necesario asumir que estos estudios de recepción deben se complementados con los de la producción por parte del museo. Pol y Asensio (1997) defienden cómo los museos evolucionaran en la medida en que lo haga la mentalidad de sus profesionales, por lo que el estudio de (sus) creencias se convierte así en una fundamental herramienta para el cambio.

Una política cultural que se fundamente en un modelo participativo supone la capacidad para identificar las posibilidades vigentes en la sociedad, para usar e interpretar el patrimonio cultural y para disponer de ese patrimonio. Por eso los estudios de las diferentes modalidades de su percepción / interpretación tanto en la instancias de la recepción del público como de la producción por los expertos no son una área solo de interés académico, son una área crucial en la gestión del patrimonio cultural y la planificación de las nuevas funciones de los museos en el mundo contemporáneo.

\section{0.- REFERENCIAS BIBLIOGRÁFICAS.}

Ballart, J. (1989). Estudios sobre Patrimonio Histórico Español. Madrid: Civitas.

Clifford, J. (2001). La interpretación de las culturas. Barcelona: Gedisa.
Comisión Nacional de Cooperación con la UNESCO (1981). Convenciones, recomendaciones y declaraciones de la UNESCO. Madrid: UNESCO.

Fernández, G. (1987). Legislación sobre Patrimonio Histórico. Madrid: Tecnos.

García, A. (1998). Didáctica del museo. El descubrimiento de los objetos. Madrid: Ediciones de la Torre.

Gauss, M. (1947). Introducción a la etnografía. Madrid: Istmo.

Morales, A. (1996). Patrimonio histórico-artístico. Madrid: Conocer el Arte. Historia16.

Piaget, J. (1982). Psicología Evolutiva. Madrid: Paidós.

Pol, E. \& Asensio, M. (1997). ¿Por qué es efectivo un montaje?: un estudio sobre las teorías de los profesionales de los museos. Boletín de ANABAD, 1, 177-195.

Prats, L. (1996). Antropología y Patrimonio. Barcelona: Mimeo.

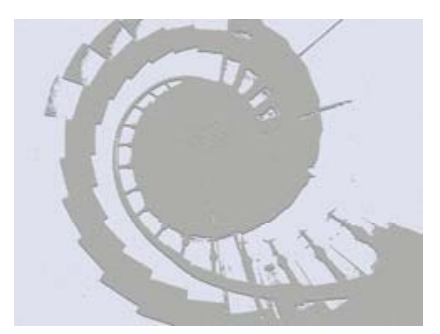

